Original

\title{
Repeatability of measurements of surface electromyographic variables during maximum voluntary contraction of temporalis and masseter muscles in normal adults
}

\author{
Yeong-Gwan Im¹), Sang-Ho Han²), Ji-Il Park'3), Hae-Soon Lim'), Byung-Gook Kim), \\ and Jae-Hyung $\mathrm{Kim}^{5)}$ \\ 1)Department of Oral Medicine, Chonnam National University Dental Hospital, Gwangju, Republic of Korea \\ ${ }^{2}$ Gns International, Daejeon, Republic of Korea \\ 3)Department of Dental Hygiene, Gwangju Health College, Gwangju, Republic of Korea \\ 4)Department of Dental Education, School of Dentistry, Chonnam National University, Gwangju, \\ Republic of Korea \\ ${ }^{5)}$ Department of Oral Medicine, School of Dentistry, Chonnam National University, Gwangju, \\ Republic of Korea
}

(Received June 2, 2016; Accepted August 22, 2016)

\begin{abstract}
This study evaluated the intraexaminer repeatability of measurements of surface electromyography (EMG) variables and functional indices of the myoelectric signals from the masseter and temporalis muscles bilaterally in $\mathbf{1 5}$ healthy men. The test was repeated on two different days without templates. The resting muscle activity was recorded once, and two kinds of maximum voluntary contraction (MVC) tasks were performed and recorded three times. The two MVC tasks involved clenching the teeth and biting down on two cotton rolls bilaterally with the posterior teeth. The intraclass correlation coefficient (ICC) of amplitude was $>\mathbf{8 8 \%}$ and that of frequency was $>95 \%$ during the two MVC tasks but not under resting conditions. The ICC of the asymmetry and activity indices during the two MVC tasks was $>76 \%$. A Bland-Altman analysis revealed no significant difference in amplitude or frequency or in the two indices between the two days during the MVC tasks. In conclusion, the measurements of surface EMG
\end{abstract}

Correspondence to Dr. Jae-Hyung Kim, Department of Oral Medicine, School of Dentistry, Chonnam National University, 33 Yongbong-ro, Buk-gu, Gwangju 61186, Republic of Korea Fax:+82-62-530-5679 E-mail: tmjkim@chonnam.ac.kr

doi.org/10.2334/josnusd.16-0434

DN/JST.JSTAGE/josnusd/16-0434 variables and the indices obtained according to the study protocol were highly repeatable in healthy men. Additional studies using templates and intraexaminer measurement errors are warranted in both men and women for complete validation of these findings.

Keywords: repeatability; surface electromyography; masseter; temporalis; maximum voluntary contraction.

\section{Introduction}

Electromyography (EMG) is a method for recording and evaluating myoelectric signals generated during muscle activation. In surface EMG, myoelectric signals are measured through the skin surface. Although surface EMG is useful to monitor the activities and understanding the function of jaw muscles, its diagnostic efficacy in disease conditions such as myalgic temporomandibular disorders is limited by numerous factors. EMG measurements may have low reliability because of technical factors, including electrode placement, electrode position, interelectrode distance, cross-talk, head or body movement, and existing pain conditions (1).

The masseter and temporalis muscles are superficially located jaw-closing muscles; thus, these are readily accessible with surface electrodes. Although these muscles are 
well isolated from other muscles, they can be influenced by cross-talk with adjacent facial muscles. Cross-talk commonly reduces the specificity of the recorded EMG signal, causing contamination by signals from nearby muscles. The myoelectric signal of these jaw muscles is most commonly acquired using a bipolar electrode configuration, wherein various interelectrode distances have been used. To reduce variability, the interelectrode distance should be fixed across different measurements, sessions, or time periods. Large interelectrode distances, up to $30 \mathrm{~mm}$, have been shown to improve the reliability of measurements of amplitude and spectral variables (2). A 20-mm interelectrode spacing or one-fourth the length of the muscle fiber was recommended for bipolar surface EMG sensors by the Surface EMG for Noninvasive Assessment of Muscles (SENIAM) group (3). Another strategy to decrease the variability related to EMG sensor repositioning involves use of a template to guide preset electrode positions in multiple examination sessions, particularly on different days (4-6).

Variability in impedance results in low reproducibility of EMG measurements. Impedance varies between active muscle fibers and electrodes and between inter- and intra-participant measurements. Several normalization procedures are available to control for inconsistent impedance. For masseter and temporalis muscles, tasks of maximum forceful tooth clenching at the intercuspal jaw position are performed to induce maximal voluntary isometric contraction as a reference value. Tasks involving biting down on the dental surfaces, cotton rolls, or other materials with maximal or submaximal effort are performed to standardize the amplitude variable and to get relevant signals of interest (7-13).

Several indices, including activity and asymmetry indices (14), have been introduced to assess coordination, synergism, and symmetry of the activities of two muscles, expanding the scope of functional analyses with surface EMG. The reliability of these indices can be ensured only when the reproducibility of raw EMG data has been validated.

Repeatability is a component of reproducibility or precision that is assessed by having a single observer performing repeated measurements on the same specimens under the same conditions. In this study, the amplitude and spectral variables of the surface EMG recordings and the activity and asymmetry indices, which were produced from the amplitude variable, were tested for repeatability or intraexaminer reproducibility in normal adults. The primary aim of this study was to investigate whether surface EMG variables and the two indices were repeatable with bipolar surface electrodes on temporalis and masseter muscles.

\section{Materials and Methods}

Participants

In total, 15 healthy men aged 25.6-36.9 (mean \pm SD: 30.3 \pm 3.9 ) years participated in this study after undergoing a screening evaluation and providing informed consent. Their mean $( \pm \mathrm{SD})$ height, weight, and body mass index (BMI) were $176.3 \pm 5.1 \mathrm{~cm}, 78.1 \pm 9.2 \mathrm{~kg}$, and $25.1 \pm 2.5$ $\mathrm{kg} / \mathrm{m}^{2}$, respectively. Participants had no tooth loss, except third molars, no fixed dental prosthesis, no crossbite, an anterior overbite of 1-5 mm, an anterior overjet of 2-5 $\mathrm{mm}$, a dental midline discrepancy of $<1 \mathrm{~mm}$, and canine guidance or group function during lateral excursive movements. Participants with known history of head injury or surgery, jaw pain, or neurological disorders were excluded. Women were excluded to control the effects of gender. Ethical approval was obtained from the Chonnam National University Dental Hospital Institutional Review Board (CNUDH-2014-006).

\section{Experimental protocol}

The same EMG examination and recording procedure was repeated on two different days less than 2 weeks apart in an isolated, quiet room containing a dental chair and under controlled illumination and temperature. The participant was seated upright in a dental chair with his head in an unsupported natural position. The participant's eyes were fixed on the wall on an object that was $1 \mathrm{~m}$ in front of him. Resting muscle activity was recorded for $30 \mathrm{~s}$ once (REST). The participant executed two kinds of maximum voluntary contraction (MVC) tasks: i) the participant was instructed to clench his jaw as strongly as possible in the intercuspal position and to maintain the maximum level of contraction for $3 \mathrm{~s}$ (MVCC), ii) the participant was instructed to bite down as strongly as possible on two $10-\mathrm{mm}$-thick cotton rolls positioned bilaterally in the premolar and molar regions for $3 \mathrm{~s}$ (MVCBB).

Three trials for each task were performed, with 30-s rest periods between trials. The two tasks were separated by a 1 -min rest period. The participant became familiar with the tasks by performing test trials before EMG recording. To standardize the contraction tasks, beep sounds were used to indicate the start and the end of the muscle contraction in each trial of the task following a preset schedule of the program (TeleScan, ver. 3.01; Laxtha, Daejeon, Korea). 


\section{Surface EMG detection}

Myoelectric signals were detected using a bipolar surface electrode configuration and a single differential EMG system. Disposable, monopolar, disc-type, $\mathrm{Ag} / \mathrm{AgCl}$ surface electrodes $10 \mathrm{~mm}$ in diameter with sticky gel (T246H, Bio Protech, Wonju, Korea) were attached to the skin after vigorous rubbing with alcohol cotton pads. Pairs of surface electrodes were located on the skin of both the left and the right superficial masseter muscles and the anterior portion of both the left and right temporalis muscles. The center-to-center distance between electrode pairs was fixed at $20 \mathrm{~mm}$ and secured with adhesive tape. A single monopolar reference electrode was placed over the skin of the spinous process of the seventh cervical vertebra.

The anterior margin of the superficial masseter belly was palpated and drawn with the participant forcefully clenching his teeth. A line, starting from the corner of the mouth and contacting the inferior border of the auricle, was drawn and defined as the first reference. Then, the intersection point of the anterior margin of the superficial masseter and the first reference line was marked. The location on the masseter muscle, one-fourth of the length from the intersection posteriorly to the contact point of the first reference line with the inferior border of the auricle, was determined and defined as a reference point. One monopolar surface electrode was placed $1 \mathrm{~cm}$ above the first reference line from the reference point, and another monopolar electrode was placed $1 \mathrm{~cm}$ below the first reference line; the two electrodes were arranged parallel to the line of the anterior margin of the superficial masseter, completing the bipolar electrode placement on the masseter muscle.

Another line, starting from the lateral canthus of the eye and contacting the superior border of the auricle, was drawn and deemed the second reference line. The location on the anterior temporalis muscle, one-fourth of the length from the lateral canthus posteriorly to the contact point of the second reference line with the superior border of the auricle, was determined for placement of one monopolar surface electrode. A line, connecting the reference point on the masseter muscle with the point for a monopolar electrode of the anterior temporalis, was drawn and defined as the third reference line. Another monopolar electrode was placed on the third reference line, $2 \mathrm{~cm}$ above the first electrode, completing the bipolar electrode placement on the anterior temporalis muscle (Fig. 1).

The signal was amplified with a $13-480-\mathrm{Hz}$ bandwidth (Butterworth filter; $-3 \mathrm{~dB}$ response; slopes of the cut-offs: low-pass $48 \mathrm{~dB}$ /octave, high-pass $12 \mathrm{~dB} /$ octave), sampled

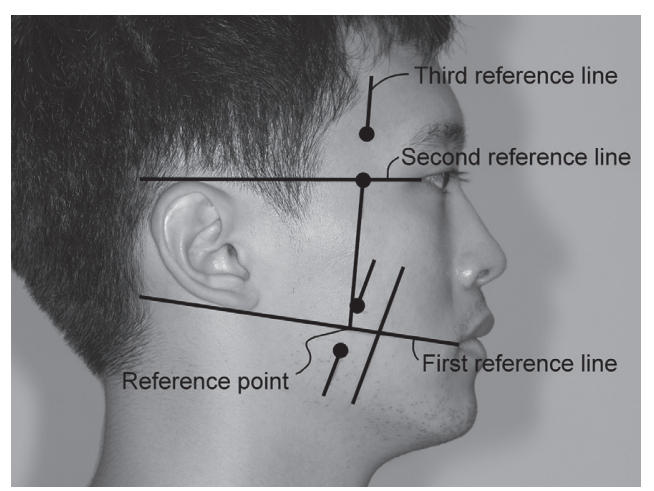

Fig. 1 Location of surface electrodes on the superficial masseter and anterior temporalis muscles

at $1024 \mathrm{~Hz}$ and digitized with a 12-bit A/D converter by using a differential amplifier with input impedance $>100$ $\mathrm{M} \Omega$, a high common mode rejection ratio (CMRR $>90$ $\mathrm{dB}$ ), and an actual gain range of 700-5,800 (LXM3204, Laxtha). Data sets were stored on the hard disk drive of a personal computer.

\section{Signal processing}

The amplitude and spectral variables were computed from the sampled signals by using TeleScan ver. 3.01 (Laxtha). The signals were digitally filtered at a band-pass range of 10-400 $\mathrm{Hz}$ and a band-stop frequency of $60 \mathrm{~Hz}$. An interval from 5-25 $\mathrm{s}$ was selected from the REST recordings, and three intervals of 2-s duration, corresponding to the first, second, and third contraction of the task trials, were selected from the MVCC and MVCBB recordings. Each interval began $1 \mathrm{~s}$ after the first beep, which indicated the beginning of a contraction, and terminated at the time of the second beep, which indicated the end of the contraction. The root-mean-square (RMS) amplitude (units: $\mu \mathrm{V}$ ) was calculated using an RMS operation function (smoothing: 200, T: 200/1024) and averaging. The median frequency (unit: $\mathrm{Hz}$ ) was calculated using a fast Fourier transform (FFT) function.

The activity index was used to compare the quantitative contributions (EMG activities) of the anterior temporalis and superficial masseter muscles to the contraction efforts (14). The activity index of the anterior temporalis and superficial masseter muscle pair was calculated for each of the right and left sides by using RMS amplitude data obtained from the two MVC tasks MVCC and MVCBB. The activity index of the anterior temporalis and superficial masseter muscle pair was defined as follows:

Activity index $\mathrm{MS}_{\mathrm{MS}}=\left(\mathrm{A}_{\mathrm{MS}}-\mathrm{A}_{\mathrm{TA}}\right) /\left(\mathrm{A}_{\mathrm{MS}}+\mathrm{A}_{\mathrm{TA}}\right) \times 100$ where $A_{M S}$ is the RMS amplitude of the superficial masseter for a specific task and $\mathrm{A}_{\mathrm{TA}}$ is that of the anterior temporalis. The activity index for the right side (AcRt) 
and that for the left side (AcLt) were calculated.

The index ranged from $-100 \%$ to $+100 \%$, with $+100 \%$ denoting exclusively superficial masseter activity. Thus, a higher activity index indicates a greater contribution from the superficial masseter muscle than from the anterior temporalis muscle.

The asymmetry index (14) was defined as follows:

Asymmetry index $=\left(\mathrm{A}_{\text {right }}-\mathrm{A}_{\text {left }}\right) /\left(\mathrm{A}_{\text {right }}+\mathrm{A}_{\text {left }}\right) \times 100$ where $A_{\text {right }}$ and $A_{\text {left }}$ are the RMS amplitudes of the right and left muscles, respectively, for a specific task. The asymmetry index for the temporalis muscle (AsTA) and that for the masseter muscle (AsMS) were calculated.

The index ranged from $-100 \%$ to $+100 \%$, with a positive index indicating right-side dominance and a negative index reflecting left-side dominance.

\section{Statistical analyses}

The reproducibility of the amplitude and frequency variables and the two indices was evaluated using the intraclass correlation coefficient (ICC), defined as follows (15):

$$
\mathrm{ICC}=100 \times \frac{\sigma_{s}^{2}}{\sigma_{t}^{2}+\sigma_{d}^{2}+\sigma_{s}^{2}}
$$

where $\sigma_{\mathrm{t}}^{2}=$ variance due to trial-to-trial variability, $\sigma_{\mathrm{d}}{ }^{2}=$ variance due to day-to-day variability, and $\sigma_{\mathrm{s}}{ }^{2}=$ variance due to inter-subject variability.

ICC is the percentage of the total variance $\left(\sigma_{\text {tot }}^{2}\right)$ due to inter-subject variability $\left(\sigma_{\mathrm{s}}^{2}\right)$. Total variance $\left(\sigma_{\text {tot }}^{2}\right)$ is the sum of the three types of variance: $\sigma_{\text {tot }}^{2}=\sigma_{\mathrm{t}}^{2}+\sigma_{\mathrm{d}}^{2}+\sigma_{\mathrm{s}}^{2}$. The percentages of variance due to trial-to-trial $\left(\sigma_{t}^{2}\right)$ and that due to day-to-day $\left(\sigma_{\mathrm{d}}^{2}\right)$ variability were computed as $100 \times{ }_{\mathrm{t}}^{2} / \sigma_{\mathrm{tot}}{ }^{2}$ and $100 \times \sigma_{\mathrm{d}}{ }^{2} / \sigma_{\mathrm{tot}}{ }^{2}$, respectively. In case of the resting condition, $\sigma_{t}^{2}$ was excluded from the equation because there was only one trial per day. ICC values $>80 \%$ suggested excellent reproducibility, those between $60 \%$ and $80 \%$ suggested good reproducibility, and those $<60 \%$ suggested poor reproducibility.

For the Bland-Altman plot analysis, the three data values obtained during the MVCC and MVCBB tasks on the first and second days were averaged to produce a mean value for each day. The Kolmogorov-Smirnov test for normality revealed that a majority of the amplitude, frequency, and index data were normally distributed, and only a few data values were not. The systematic error between the measurements of the two consecutive days was examined using the mean of the differences with the $95 \%$ confidence interval (CI). Limits of agreement (LoAs) or $95 \%$ CIs were calculated as the mean difference \pm 1.96 standard deviations, which was depicted in the Bland-Altman plot (16).

ICC was statistically analyzed using "R" (ver. 3.2.2), and the other analyses were performed using the SPSS software (ver. 21.0; SPSS Inc., Chicago, IL, USA).

\section{Results}

ICCs of the RMS amplitude variable of all muscle sites during the MVCC and MVCBB tasks were $>88 \%$, and those of the median frequency variable were $>95 \%$ during the two tasks, indicating excellent reproducibility. Under the resting condition, ICCs of the amplitude of the masseter muscle of the right side (RtMS) and of the left side (LtMS) were approximately 94\%, whereas those of the temporalis muscle of the right side (RtTA) and of the left side (LtTA) were approximately $50 \%$. ICCs of the median frequency variable under the resting condition were more variable. The ICC of the frequency of LtTA was even negative, with high day-to-day variance $\left(\sigma_{d}^{2}\right)$. ICCs of the asymmetry index during the MVCC and MVCBB tasks (AsTA and AsMS) were $>95 \%$, indicating excellent reproducibility. ICCs of the activity index during the two tasks AcRt and AcLt were between $76 \%$ and $89 \%$, indicating good-to-excellent reproducibility (Table 1).

The mean differences in all amplitude and frequency variables and the two indices were small, and the $95 \%$ LoA for all variables and indices were narrow, indicating good repeatability between the two days for the two MVC tasks. For example, the mean differences in the median frequency variable for the two MVC tasks were extremely small, considering its day mean values, varying between -2.0 and 2.0 (Table 2). Bland-Altman plots indicated no significant difference in the variables or indices between the two days for the two MVC tasks; most values were within the limits of the 95\% CI (Figs. 2-5).

However, under the resting condition, relatively high mean differences and wider 95\% LoAs were noted in the amplitude data of the anterior temporalis muscle and the median frequency data of the anterior temporalis and masseter muscles. For example, the mean difference in the median frequency variable of the LtTA under the resting condition was largest, at 27.6, with 95\% LoAs ranging from -93.49 to 148.70 . The mean differences in the asymmetry index during the MVCC and MVCBB tasks ranged from -1.0 to 1.0 , and those in the activity index during the two MVC tasks ranged from 1.88 to 3.02 (Table 2).

\section{Discussion}

The present study confirmed an extremely high level of reproducibility with high ICC values and narrow $95 \%$ LoAs for the amplitude and spectral variable data and the 
Table 1 Intraclass correlation coefficient (ICC) and percent of variance due to day-to-day $\left(\sigma_{d}^{2}\right)$ and trial-to-trial $\left(\sigma_{t}^{2}\right)$ variability for the RMS amplitude variable, the median frequency variable, and the asymmetry and activity indices

\begin{tabular}{|c|c|c|c|c|c|c|c|c|c|c|c|c|}
\hline \multirow[b]{2}{*}{ Task } & \multicolumn{4}{|c|}{ RMS amplitude $(\mu \mathrm{V})$} & \multicolumn{4}{|c|}{ Median frequency $(\mathrm{Hz})$} & \multicolumn{4}{|c|}{ Asymmetry and activity indices (\%) } \\
\hline & Muscle & ICC & $\sigma_{d}^{2}$ & $\sigma_{t}^{2}$ & Muscle & ICC & $\sigma_{d}^{2}$ & $\sigma_{t}^{2}$ & Index & ICC & $\sigma_{d}^{2}$ & $\sigma_{t}^{2}$ \\
\hline \multirow[t]{4}{*}{ REST } & RtTA & 51.49 & 48.51 & - & RtTA & 85.09 & 14.91 & - & & & & \\
\hline & LtTA & 49.18 & 50.82 & - & LtTA & -28.11 & 128.11 & - & & & & \\
\hline & RtMS & 93.97 & 6.03 & - & RtMS & 49.80 & 50.20 & - & & & & \\
\hline & LtMS & 93.58 & 6.42 & - & LtMS & 31.59 & 68.41 & - & & & & \\
\hline \multirow[t]{4}{*}{ MVCC } & RtTA & 92.65 & 6.72 & 0.63 & RtTA & 96.89 & 2.27 & 0.84 & AsTA & 95.63 & 3.16 & 1.21 \\
\hline & LtTA & 88.50 & 9.91 & 1.58 & LtTA & 96.66 & 3.29 & 0.05 & AsMS & 98.65 & 1.21 & 0.14 \\
\hline & RtMS & 96.74 & 2.15 & 1.11 & RtMS & 99.79 & -0.08 & 0.29 & AcRt & 88.96 & 10.94 & 0.10 \\
\hline & LtMS & 98.55 & -0.04 & 1.48 & LtMS & 98.65 & -0.64 & 1.99 & AcLt & 76.01 & 23.56 & 0.43 \\
\hline \multirow[t]{4}{*}{ MVCBB } & RtTA & 99.27 & 0.34 & 0.39 & RtTA & 98.03 & 1.64 & 0.34 & AsTA & 98.61 & 1.18 & 0.21 \\
\hline & LtTA & 94.67 & 4.02 & 1.31 & LtTA & 97.43 & 1.08 & 1.49 & AsMS & 96.10 & -1.57 & 5.47 \\
\hline & RtMS & 91.41 & 8.44 & 0.15 & RtMS & 96.48 & -1.00 & 4.52 & AcRt & 80.82 & 16.54 & 2.65 \\
\hline & LtMS & 93.17 & 4.48 & 2.35 & LtMS & 95.34 & 0.15 & 4.51 & AcLt & 76.18 & 22.72 & 1.09 \\
\hline
\end{tabular}

$n=15$. REST: resting jaw condition, MVCC: maximum jaw clenching in the intercuspal position, MVCBB: maximum bilateral biting of two cotton rolls with the posterior teeth, RtTA: right anterior temporalis, LtTA: left anterior temporalis, RtMS: right superficial masseter, LtMS: left superficial masseter, AsTA: asymmetry index for the temporalis muscle, AsMS: asymmetry index for the masseter muscle, AcRt: activity index for the right side, AcLt: activity index for the left side.

Table 2 Mean (SD) values and mean of the differences in the RMS amplitude variable, median frequency variable, and asymmetry and activity indices for each muscle on the two consecutive days

\begin{tabular}{|c|c|c|c|c|c|c|c|c|c|c|c|c|}
\hline \multirow[b]{2}{*}{ Task } & \multicolumn{4}{|c|}{ RMS amplitude $(\mu \mathrm{V})$} & \multicolumn{4}{|c|}{ Median frequency $(\mathrm{Hz})$} & \multicolumn{4}{|c|}{ Asymmetry and activity indices (\%) } \\
\hline & Muscle & $\begin{array}{c}\text { Day } 1 \\
\text { mean (SD) }\end{array}$ & $\begin{array}{c}\text { Day } 2 \\
\text { mean (SD) }\end{array}$ & $\begin{array}{c}\text { Mean } \\
\text { difference } \\
(95 \% \mathrm{CI})\end{array}$ & Muscle & $\begin{array}{c}\text { Day } 1 \\
\text { mean (SD) }\end{array}$ & $\begin{array}{c}\text { Day } 2 \\
\text { mean }(\mathrm{SD})\end{array}$ & $\begin{array}{c}\text { Mean } \\
\text { difference } \\
(95 \% \mathrm{CI})\end{array}$ & Index & $\begin{array}{c}\text { Day } 1 \\
\text { mean }(\mathrm{SD})\end{array}$ & $\begin{array}{c}\text { Day } 2 \\
\text { mean }(\mathrm{SD})\end{array}$ & $\begin{array}{c}\text { Mean } \\
\text { difference } \\
(95 \% \mathrm{CI})\end{array}$ \\
\hline \multirow[t]{4}{*}{ REST } & RtTA & $\begin{array}{r}2.56 \\
(0.67)\end{array}$ & $\begin{array}{r}2.80 \\
(1.00)\end{array}$ & $\begin{array}{c}-0.25 \\
(2.12-2.61)\end{array}$ & RtTA & $\begin{array}{r}93.0 \\
(36.9)\end{array}$ & $\begin{array}{r}87.6 \\
(33.9)\end{array}$ & $\begin{array}{c}5.40 \\
(99.35-88.54)\end{array}$ & & & & \\
\hline & LtTA & $\begin{array}{r}2.88 \\
(0.90)\end{array}$ & $\begin{array}{r}2.64 \\
(0.62)\end{array}$ & $\begin{array}{c}0.24 \\
(2.31-1.83)\end{array}$ & LtTA & $\begin{array}{l}121.2 \\
(46.4)\end{array}$ & $\begin{array}{r}93.6 \\
(36.9)\end{array}$ & $\begin{array}{c}27.60 \\
(148.70-93.49)\end{array}$ & & & & \\
\hline & RtMS & $\begin{array}{r}2.05 \\
(0.30)\end{array}$ & $\begin{array}{r}2.09 \\
(0.41)\end{array}$ & $\begin{array}{c}-0.04 \\
(0.76-0.84)\end{array}$ & RtMS & $\begin{array}{l}111.9 \\
(59.9)\end{array}$ & $\begin{array}{r}90.3 \\
(53.2)\end{array}$ & $\begin{array}{c}21.58 \\
(117.99-74.84)\end{array}$ & & & & \\
\hline & LtMS & $\begin{array}{r}2.00 \\
(0.35)\end{array}$ & $\begin{array}{r}1.97 \\
(0.35)\end{array}$ & $\begin{array}{c}0.03 \\
(0.97-0.91)\end{array}$ & LtMS & $\begin{array}{l}104.7 \\
(51.8)\end{array}$ & $\begin{array}{r}81.7 \\
(50.8)\end{array}$ & $\begin{array}{c}22.98 \\
(128.68-82.72)\end{array}$ & & & & \\
\hline \multirow[t]{4}{*}{ MVCC } & RtTA & $\begin{array}{l}143.87 \\
(69.92)\end{array}$ & $\begin{array}{l}153.39 \\
(68.45)\end{array}$ & $\begin{array}{c}-9.51 \\
(26.81-45.84)\end{array}$ & RtTA & $\begin{array}{l}161.57 \\
(21.43)\end{array}$ & $\begin{array}{l}163.30 \\
(21.19)\end{array}$ & $\begin{array}{c}-1.73 \\
(16.48-19.95)\end{array}$ & AsTA & $\begin{array}{c}3.15 \\
(10.00)\end{array}$ & $\begin{array}{r}2.27 \\
(9.19)\end{array}$ & $\begin{array}{c}0.88 \\
(15.10-13.35)\end{array}$ \\
\hline & LtTA & $\begin{array}{l}135.58 \\
(61.25)\end{array}$ & $\begin{array}{l}145.64 \\
(57.06)\end{array}$ & $\begin{array}{c}-10.06 \\
(29.67-49.79)\end{array}$ & LtTA & $\begin{array}{l}164.77 \\
(21.21)\end{array}$ & $\begin{array}{l}162.89 \\
(19.76)\end{array}$ & $\begin{array}{c}1.88 \\
(22.21-18.45)\end{array}$ & AsMS & $\begin{array}{l}-1.04 \\
(11.36)\end{array}$ & $\begin{array}{l}-0.41 \\
(12.02)\end{array}$ & $\begin{array}{c}-0.64 \\
(14.79-16.07)\end{array}$ \\
\hline & RtMS & $\begin{array}{c}275.52 \\
(124.37)\end{array}$ & $\begin{array}{c}285.20 \\
(112.12)\end{array}$ & $\begin{array}{c}-9.67 \\
(69.90-89.24)\end{array}$ & RtMS & $\begin{array}{l}146.96 \\
(17.84)\end{array}$ & $\begin{array}{l}146.83 \\
(15.70)\end{array}$ & $\begin{array}{c}0.12 \\
(9.97-9.73)\end{array}$ & AcRt & $\begin{array}{l}32.05 \\
(11.27)\end{array}$ & $\begin{array}{c}30.17 \\
(10.90)\end{array}$ & $\begin{array}{c}1.88 \\
(13.66-9.90)\end{array}$ \\
\hline & LtMS & $\begin{array}{c}285.88 \\
(136.75)\end{array}$ & $\begin{array}{c}290.39 \\
(123.11)\end{array}$ & $\begin{array}{c}-4.51 \\
(85.13-94.15)\end{array}$ & LtMS & $\begin{array}{l}155.10 \\
(21.80)\end{array}$ & $\begin{array}{l}155.27 \\
(18.38)\end{array}$ & $\begin{array}{c}-0.17 \\
(15.11-15.44)\end{array}$ & AcLt & $\begin{array}{c}35.62 \\
(12.68)\end{array}$ & $\begin{array}{c}32.61 \\
(11.33)\end{array}$ & $\begin{array}{c}3.02 \\
(19.60-13.57)\end{array}$ \\
\hline \multirow[t]{4}{*}{ MVCBB } & RtTA & $\begin{array}{l}135.73 \\
(56.25)\end{array}$ & $\begin{array}{l}137.71 \\
(56.91)\end{array}$ & $\begin{array}{c}-1.98 \\
(40.98-44.95)\end{array}$ & RtTA & $\begin{array}{l}156.86 \\
(23.29)\end{array}$ & $\begin{array}{l}158.39 \\
(22.54)\end{array}$ & $\begin{array}{c}-1.53 \\
(18.74-21.81)\end{array}$ & AsTA & $\begin{array}{r}1.23 \\
(7.52)\end{array}$ & $\begin{array}{r}0.80 \\
(9.50)\end{array}$ & $\begin{array}{c}0.44 \\
(16.22-15.34)\end{array}$ \\
\hline & LtTA & $\begin{array}{l}129.69 \\
(41.84)\end{array}$ & $\begin{array}{l}134.63 \\
(50.38)\end{array}$ & $\begin{array}{c}-4.94 \\
(44.39-54.27)\end{array}$ & LtTA & $\begin{array}{l}158.64 \\
(20.13)\end{array}$ & $\begin{array}{l}157.49 \\
(16.11)\end{array}$ & $\begin{array}{c}1.16 \\
(19.43-17.12)\end{array}$ & AsMS & $\begin{array}{c}0.72 \\
(10.67)\end{array}$ & $\begin{array}{c}1.02 \\
(11.86)\end{array}$ & $\begin{array}{c}-0.29 \\
(11.01-11.60)\end{array}$ \\
\hline & RtMS & $\begin{array}{l}287.27 \\
(95.38)\end{array}$ & $\begin{array}{l}274.38 \\
(81.46)\end{array}$ & $\begin{array}{c}12.89 \\
(127.38- \\
101.60)\end{array}$ & RtMS & $\begin{array}{l}140.39 \\
(16.50)\end{array}$ & $\begin{array}{l}141.04 \\
(19.14)\end{array}$ & $\begin{array}{c}-0.66 \\
(15.06-16.37)\end{array}$ & AcRt & $\begin{array}{c}36.47 \\
(10.61)\end{array}$ & $\begin{array}{l}34.29 \\
(9.73)\end{array}$ & $\begin{array}{c}2.18 \\
(15.89-11.53)\end{array}$ \\
\hline & LtMS & $\begin{array}{c}287.17 \\
(104.75)\end{array}$ & $\begin{array}{c}274.81 \\
(101.57)\end{array}$ & $\begin{array}{c}12.36 \\
(74.82-50.09)\end{array}$ & LtMS & $\begin{array}{l}150.44 \\
(16.93)\end{array}$ & $\begin{array}{l}149.28 \\
(18.77)\end{array}$ & $\begin{array}{c}1.17 \\
(20.92-18.59)\end{array}$ & AcLt & $\begin{array}{c}36.93 \\
(11.08)\end{array}$ & $\begin{array}{c}33.93 \\
(12.10)\end{array}$ & $\begin{array}{c}2.99 \\
(13.50-7.52)\end{array}$ \\
\hline
\end{tabular}

$n=15.95 \%$ CI: 95 percent confidence interval, REST: resting jaw condition, MVCC: maximum jaw clenching in the intercuspal position, MVCBB: maximum bilateral biting of two cotton rolls with the posterior teeth, RtTA: right anterior temporalis, LtTA: left anterior temporalis, RtMS: right superficial masseter, LtMS: left superficial masseter, AsTA: asymmetry index for the temporalis muscle, AsMS: asymmetry index for the masseter muscle, AcRt: activity index for the right side, AcLt: activity index for the left side. 

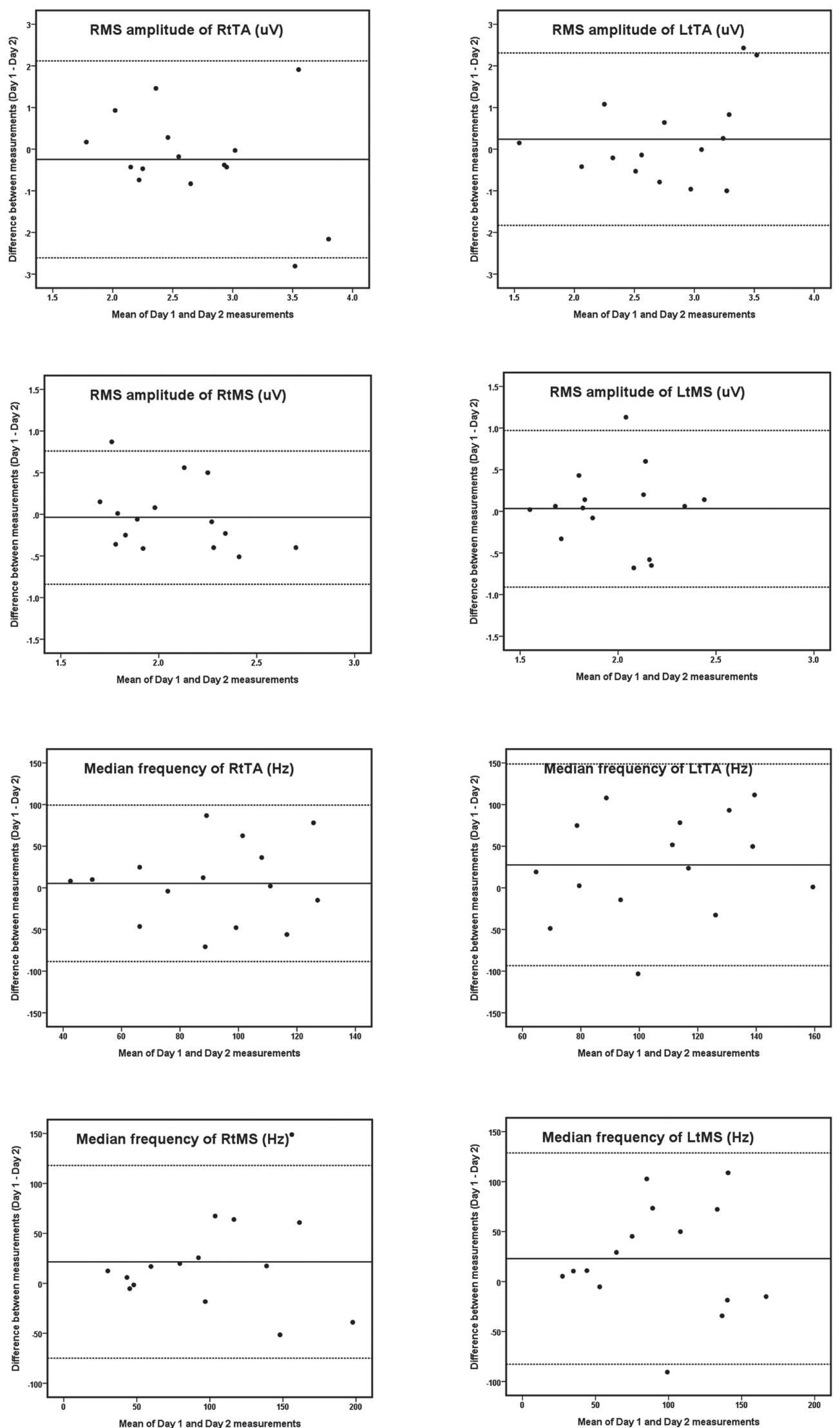

Fig. 2 Bland-Altman plots for amplitude and power spectrum frequency variables of the myoelectric signals from the temporalis and masseter muscles on the right and left sides under the resting condition for the two consecutive days. RMS: root mean square, REST: resting jaw condition, RtTA: right anterior temporalis, LtTA: left anterior temporalis, RtMS: right superficial masseter, LtMS: left superficial masseter. 

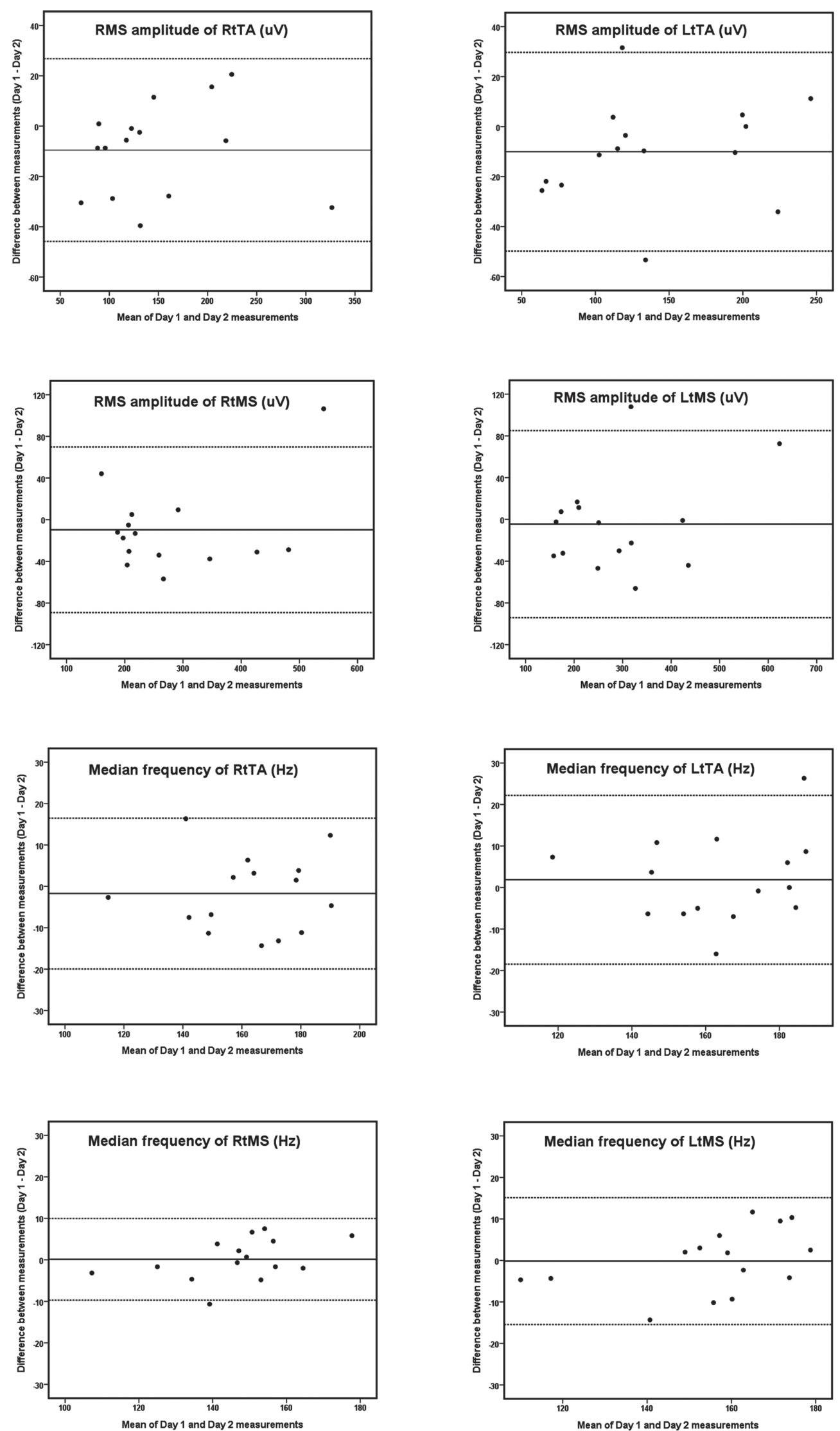

Fig. 3 Bland-Altman plots for amplitude and power spectrum frequency variables of the myoelectric signals from the temporalis and masseter muscles on the right and left sides during the MVCC task for the two consecutive days. RMS: root mean square, MVCC: maximum jaw clenching in the intercuspal position, RtTA: right anterior temporalis, LtTA: left anterior temporalis, RtMS: right superficial masseter, LtMS: left superficial masseter. 

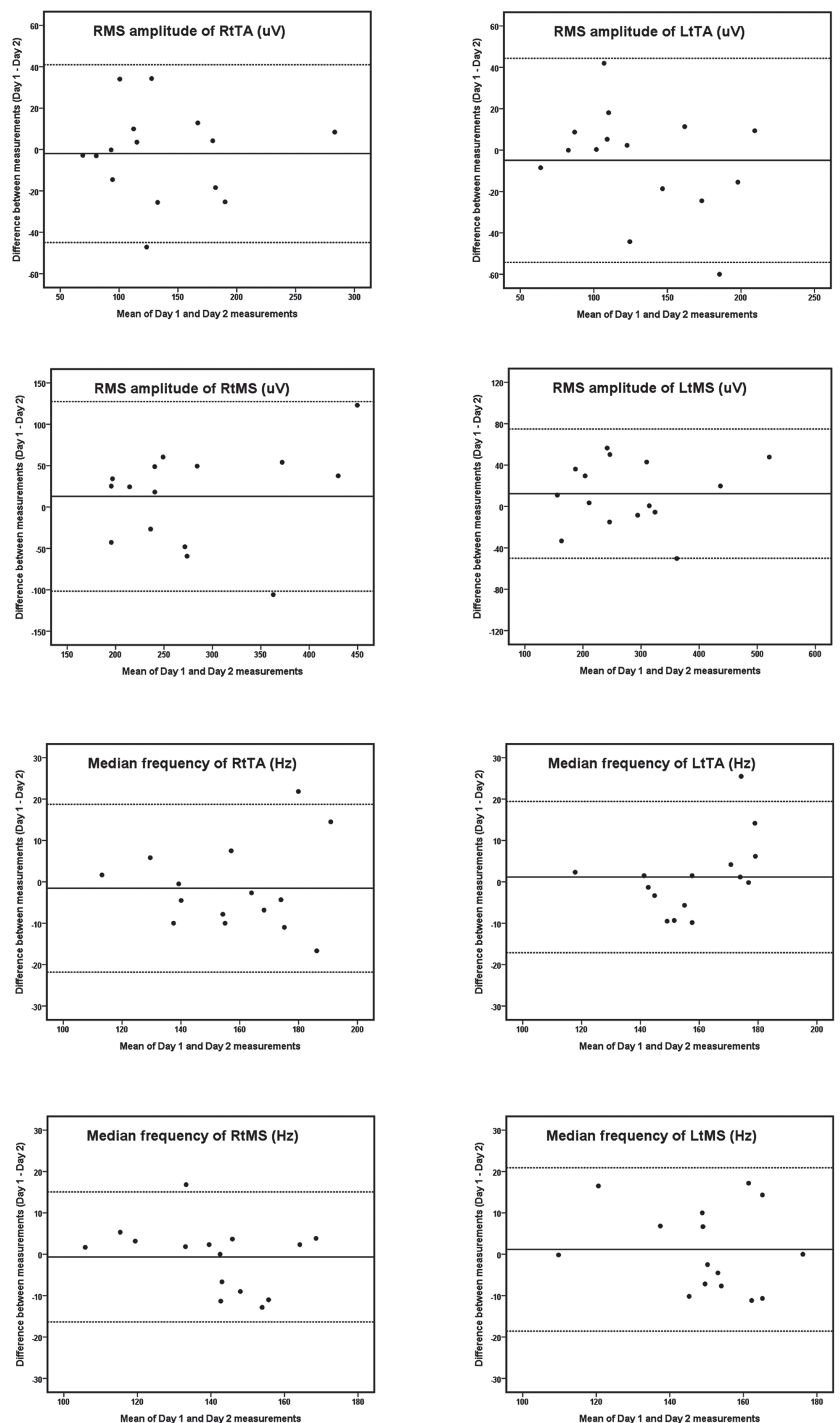

Fig. 4 Bland-Altman plots for amplitude and power spectrum frequency variables of the myoelectric signals from the temporalis and masseter muscles on the right and left sides during the MVCBB task for the two consecutive days. RMS: root mean square, MVCBB: maximum bilateral biting of two cotton rolls with the posterior teeth, RtTA: right anterior temporalis, LtTA: left anterior temporalis, RtMS: right superficial masseter, LtMS: left superficial masseter. 

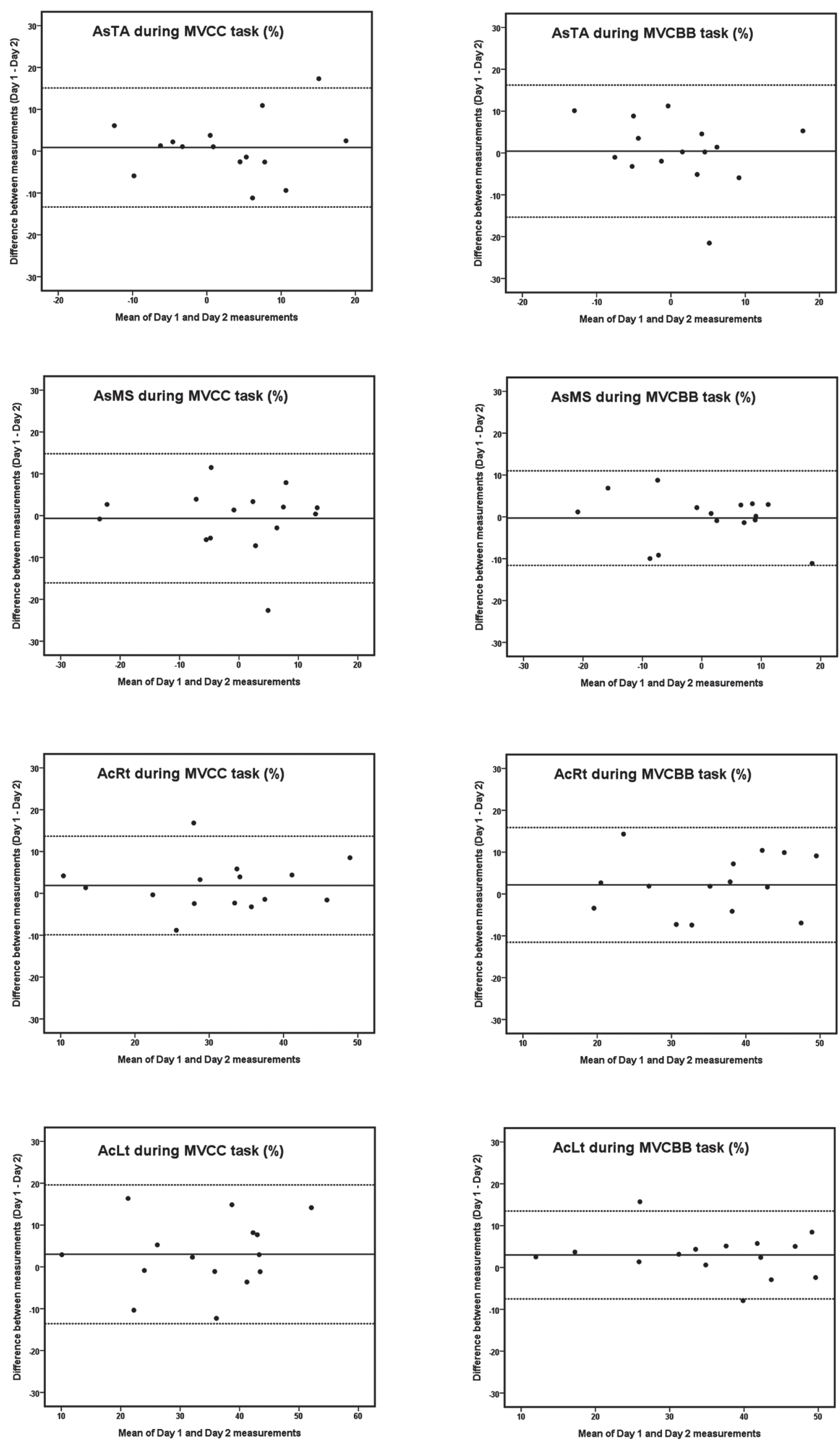

Fig. 5 Bland-Altman plots for asymmetry and activity indices of the myoelectric signals from the temporalis and masseter muscles during the MVCC and MVCBB tasks for the two consecutive days. MVCC: maximum jaw clenching in the intercuspal position, MVCBB: maximum bilateral biting of two cotton rolls with the posterior teeth, AsTA: asymmetry index for the temporalis muscle, AsMS: asymmetry index for the masseter muscle, AcRt: activity index for the right side, AcLt: activity index for the left side. 
two indices calculated from the amplitude during the two MVC tasks, obtained by following the protocol described for the anterior temporalis and superficial masseter muscles in healthy men.

ICCs of the RMS amplitude and median frequency for the two MVC tasks ranged between $88 \%$ and $99 \%$, indicating excellent reproducibility. The high reproducibility of surface EMG variables during MVC tasks is consistent with previous studies (6,17-19). Specifically, ICCs of the median frequency variable were $>95 \%$, suggesting greater reproducibility than that of the amplitude variable. This finding corresponds well with that of a previous study (6). The reproducibility of EMG amplitudes is known to be the highest for those obtained during MVC activities. For example, at an $80 \%$ MVC force level, ICCs of the average rectified value and mean power spectral frequency with an interelectrode distance of $20 \mathrm{~mm}$ were $<80 \%$ (2), suggesting good, but not excellent, reproducibility.

The reproducibility of the RMS amplitude of the masseter muscle under the resting condition was also excellent, with ICCs around 94\%, which corresponded well with those used in a previous study (4). In contrast, the ICC of the amplitude of the anterior temporalis muscle was approximately $50 \%$, and its $95 \%$ LoA was wide, considering the mean values for each day, indicating poor reproducibility and poor agreement between days. Greater variability among repeated measurements of the resting EMG amplitude, particularly of the anterior temporalis muscle, was reported in patients with myalgia (4). In healthy adults, repeated EMG amplitude recordings of the masseter and anterior temporalis muscles in the mandibular rest position were reliable within the same session (5) but significantly different on different days $(5,6)$.

The excellent repeatability of the median frequency during the MVC tasks of this study is consistent with findings from a previous study (6). In contrast, ICCs of the median frequency under the resting condition were variable with poor repeatability; those of the masseter muscle were $<50 \%$, whereas those of the left temporalis muscle were even negative. Bland-Altman analysis indicated poor agreement of the median frequency data between the two days, showing large mean differences and a wide $95 \%$ LoA. Highly variable data on repeated measurements, such as the median frequency under the resting condition, are unreliable and thus clinically unacceptable. The low repeatability of surface EMG variables recorded under the resting condition may be related to methodological errors across different trials and sessions as well as the dynamic response of the muscle to experi- mental conditions.

The negative ICC of the left temporalis muscle is perplexing, but negative values can be generated when there is little variability among participants or considerable variability between days (for a more detailed explanation, see Reinoldi et al. [20]). The negative ICC of the LtTA frequency was associated with high day-to-day variance, which was also confirmed in the Bland-Altman plot analysis, which showed a large mean difference and a wide LoA between the two consecutive days. In this study, the same EMG recording procedure was repeated on two different days less than 2 weeks apart. We made an effort to perform the second EMG recording as soon as possible after the first, without exceeding 1 week; however, certain participants could not meet this schedule. Intervals between a test and a retest in previous surface EMG studies for the temporalis and masseter muscles reportedly varied by 1 day (19), 1 week $(2,5), 2$ weeks $(4,7)$, or 6 months (17).

Reproducibility of the asymmetry and activity indices was good to excellent, with ICCs ranging from 76 to $99 \%$. This was consistent with previous reports of good reproducibility of several indices of symmetry, torque coefficient, relative activity, and total activity of the temporalis and masseter muscles $(17,19)$. It is noteworthy that ICCs of the asymmetry index were greater than those of the activity index, indicating that the asymmetry index is more reproducible than the activity index, although both were highly reproducible during the MVC tasks.

This study introduced a method of positioning surface electrodes on the superficial masseter and anterior temporalis muscles based on anatomical landmarks of the face for a single differential amplification system. For the superficial masseter muscle, a pair of monopolar surface electrodes was placed parallel to the traced line of the anterior margin of the muscle. The anterior margin of the superficial masseter is easily palpable when a participant clenches his teeth. It is reasonable to suppose that the direction of the pair of two monopolar surface electrodes is parallel to that of the underlying muscle fibers of the superficial masseter. The pair of surface electrodes was placed one-fourth of the length posteriorly from the intersection to the contact point of the first reference line with the inferior border of the auricle, which was 1-1.5 $\mathrm{cm}$ posterior to the anterior margin of the superficial masseter in men. This anteroposterior position of the electrode pair seems adequate because it is located far enough from the adjacent facial muscles anteriorly and from the parotid gland posteriorly. Myoelectric signals detected by surface EMG sensors are primarily from muscle fibers within 10-12 mm (21). Consequently, the 
surface EMG recordings from this position should represent exclusive activity of the masseter muscle.

Using manual palpation, we repeatedly found that the anteroposterior position of the reference point on the superficial masseter region corresponded well with that of the anterior border of the coronoid process of the mandible in several participants. The temporalis muscle is attached to the surface of the coronoid process of the mandible and along the anterior border of the mandibular ramus. Thus, the third reference line, which connects the reference point on the masseter with the position of the first monopolar surface electrode for the anterior temporalis muscle, is assumed to run parallel to the fiber direction of the anterior temporalis muscle. Then, the position of the second monopolar surface electrode is determined above the first one on the third reference line to complete the bipolar configuration. The anteroposterior position of the first electrode was arbitrarily defined at one-fourth of the length posteriorly from the lateral canthus to the contact point of the second reference line with the superior border of the auricle. We thought this position was convenient and appropriate because it was $>1 \mathrm{~cm}$ away from the anterior border of the temporalis belly and anterior to the hairline of the temporal region in most men.

The superoinferior position of the surface electrodes was defined using the two reference lines that were, in part, related to the external ear structure. Although the shape and size of the ear is affected by age, gender, and ethnic factors, the three-dimensional position of the right and left ears is symmetrical (22), and most measurements between the two sides are also highly symmetrical (23). These anatomical features of the ear contribute to the symmetrical positioning of surface electrodes on the masseter and anterior temporalis muscles. However, the exact superoinferior position of the surface electrodes for the superficial masseter and anterior temporalis muscles is still arbitrary. It is generally recommended that the bipolar surface EMG electrodes be applied between the innervation zone and the tendon area and that both electrodes should be on one side of the innervation zone (24). The innervation zones of the superficial masseter and anterior temporalis muscles are scattered in large regions up to approximately $50 \%$ of the fiber length; thus, the optimal position of the surface EMG electrodes for these muscles could not be determined (25). In another study, the neuromuscular junctions of both the superficial masseter and temporalis muscles were distributed within $10 \mathrm{~mm}$ along the muscle fibers, which were located in the lower part of the masseter and in the upper part of the temporalis muscle (26). With regard to the masseter muscle, the lower half, except the end-plate zone, which was found in the middle of the lower half of the muscle, was recommended for surface EMG recording (27).

The SENIAM group recommended an interelectrode spacing of $20 \mathrm{~mm}$ or one-fourth the length of the muscle fiber (3). The fiber lengths of the superficial masseter and anterior temporalis were measured at 25 and $27 \mathrm{~mm}$, respectively, in an anatomical study (28), and these were estimated at 27 and $26 \mathrm{~mm}$, respectively, with surface EMG (25). One-fourth of these data ranged 6-7 $\mathrm{mm}$. This length is not preferred for a bipolar electrode configuration owing to the size of the regular surface electrodes. However, larger electrodes and interelectrode distances improve the reproducibility of measurements $(5,10)$, and an interelectrode distance of at least 10-15 $\mathrm{mm}$ has been suggested (10). We used disc-type monopolar surface electrodes that were $10 \mathrm{~mm}$ in diameter. The interelectrode distance of the paired single surface electrodes was fixed at $20 \mathrm{~mm}$ by means of an adhesive tape. The interelectrode distance of $20 \mathrm{~mm}$ adopted in our study has the advantage of enabling comparisons of data from several different studies by using this distance because $20 \mathrm{~mm}$ is the most preferred distance.

Cross-talk is the EMG signal generated by nearby muscles and transmitted via volume conduction to the recording electrode. The recorded low-voltage EMG signal from the anterior temporalis muscle in the rest position could be affected by activity from other muscles in healthy participants when their eyes are open (29). In contrast, another recent study reported no significant difference in the amplitude of the anterior temporalis muscle in the mandibular rest position between closed-and open-eye conditions in young, healthy participants (30). Cross-talk signals have different shape and frequency components compared with the EMG signals detected over the active muscle; thus, cross-talk signals can be interpreted, quantified, and reduced if their origins are clearly determined (31).

The findings of this study are limited for the following reasons. First, one independent researcher participated in the test, and consequently, inter-rater reproducibility was not evaluated. Second, the amplitude and spectral variables were tested only for MVC tasks, with no absolute muscle contraction force or biting force measurements. Various submaximal contraction activities might provide additional information. Third, templates were not used to locate the surface EMG sensors across different days; otherwise, it might have been possible to increase the repeatability of the measurements. Finally, this method of locating surface electrodes on the masseter and temporalis muscles may not be appropriate for participants with 
different craniofacial dimensions from those of adult men or in individuals with ear deformities.

In conclusion, this study revealed high repeatability of measurements of amplitude and spectral variables as well as the two indices obtained according to the protocol on the anterior temporalis and superficial masseter muscles during MVC tasks performed by healthy men. The variability in data between repeated measurements was small, and the data can be considered reliable for clinical use. However, these high-repeatability results should be interpreted within these limitations, and additional studies must be conducted using templates and intraexaminer measurement errors in both men and women for complete validation of these findings.

\section{Conflict of interest}

No conflict of interest has been reported by the authors or by any individual in control of the content of this article.

\section{References}

1. Klasser GD, Okeson JP (2006) The clinical usefulness of surface electromyography in the diagnosis and treatment of temporomandibular disorders. J Am Dent Assoc 137, 763-771.

2. Castroflorio T, Icardi K, Becchino B, Merlo E, Debernardi C, Bracco P et al. (2006) Reproducibility of surface EMG variables in isometric sub-maximal contractions of jaw elevator muscles. J Electromyogr Kinesiol 16, 498-505.

3. Hermens HJ, Freriks B, Disselhorst-Klug C, Rau G (2000) Development of recommendations for SEMG sensors and sensor placement procedures. J Electromyogr Kinesiol 10, 361-374.

4. Burdette BH, Gale EN (1990) Reliability of surface electromyography of the masseteric and anterior temporal areas. Arch Oral Biol 35, 747-751.

5. Castroflorio T, Icardi K, Torsello F, Deregibus A, Debernardi C, Bracco P (2005) Reproducibility of surface EMG in the human masseter and anterior temporalis muscle areas. Cranio 23, 130-137.

6. Suvinen TI, Malmberg J, Forster C, Kemppainen P (2009) Postural and dynamic masseter and anterior temporalis muscle EMG repeatability in serial assessments. J Oral Rehabil 36, 814-820.

7. Ferrario VF, Sforza C, D’Addona A, Miani A Jr (1991) Reproducibility of electromyographic measures: a statistical analysis. J Oral Rehabil 18, 513-521.

8. Ferrario VF, Sforza C, Colombo A, Ciusa V (2000) An electromyographic investigation of masticatory muscles symmetry in normo-occlusion subjects. J Oral Rehabil 27, 33-40.

9. Saifuddin M, Miyamoto K, Ueda HM, Shikata N, Tanne K (2001) A quantitative electromyographic analysis of masticatory muscle activity in usual daily life. Oral Dis 7, 94-100.
10. Castroflorio T, Farina D, Bottin A, Piancino MG, Bracco P, Merletti R (2005) Surface EMG of jaw elevator muscles: effect of electrode location and inter-electrode distance. J Oral Rehabil 32, 411-417.

11. Ferrario VF, Tartaglia GM, Galletta A, Grassi GP, Sforza C (2006) The influence of occlusion on jaw and neck muscle activity: a surface EMG study in healthy young adults. J Oral Rehabil 33, 341-348.

12. Botelho AL, Gentil FH, Sforza C, da Silva MA (2011) Standardization of the electromyographic signal through the maximum isometric voluntary contraction. Cranio 29, 23-31.

13. Lodetti G, Mapelli A, Musto F, Rosati R, Sforza C (2012) EMG spectral characteristics of masticatory muscles and upper trapezius during maximum voluntary teeth clenching. $\mathrm{J}$ Electromyogr Kinesiol 22, 103-109.

14. Naeije M, McCarroll RS, Weijs WA (1989) Electromyographic activity of the human masticatory muscles during submaximal clenching in the inter-cuspal position. J Oral Rehabil 16, 63-70.

15. Bartko JJ (1966) The intraclass correlation coefficient as a measure of reliability. Psychol Rep 19, 3-11.

16. Bland JM, Altman DG (1986) Statistical methods for assessing agreement between two methods of clinical measurement. Lancet 1, 307-310.

17. De Felício CM, Sidequersky FV, Tartaglia GM, Sforza C (2009) Electromyographic standardized indices in healthy Brazilian young adults and data reproducibility. J Oral Rehabil 36, 577-583.

18. Forrester SE, Allen SJ, Presswood RG, Toy AC, Pain MT (2010) Neuromuscular function in healthy occlusion. J Oral Rehabil 37, 663-669.

19. Sforza C, Rosati R, De Menezes M, Musto F, Toma M (2011) EMG analysis of trapezius and masticatory muscles: experimental protocol and data reproducibility. J Oral Rehabil 38, 648-654.

20. Rainoldi A, Galardi G, Maderna L, Comi G, Lo Conte L, Merletti R (1999) Repeatability of surface EMG variables during voluntary isometric contractions of the biceps brachii muscle. J Electromyogr Kinesiol 9, 105-119.

21. Fuglevand AJ, Winter DA, Patla AE, Stashuk D (1992) Detection of motor unit action potentials with surface electrodes: influence of electrode size and spacing. Biol Cybern 67, 143-153.

22. Sforza C, Grandi G, Binelli M, Tommasi DG, Rosati R, Ferrario VF (2009) Age- and sex-related changes in the normal human ear. Forensic Sci Int 187, 110.e1-7.

23. Alexander KS, Stott DJ, Sivakumar B, Kang N (2011) A morphometric study of the human ear. J Plast Reconstr Aesthet Surg 64, 41-47.

24. Mesin L, Merletti R, Rainoldi A (2009) Surface EMG: the issue of electrode location. J Electromyogr Kinesiol 19, 719-726.

25. Castroflorio T, Farina D, Bottin A, Debernardi C, Bracco P, Merletti R et al. (2005) Non-invasive assessment of motor unit anatomy in jaw-elevator muscles. J Oral Rehabil 32, 
708-713.

26. Tokunaga T, Baba S, Tanaka M, Kashiwagi K, Kimura K, Kawazoe T (1998) Two-dimensional configuration of the myoneural junctions of human masticatory muscle detected with matrix electrode. J Oral Rehabil 25, 329-334.

27. Iwasaki S, Tokunaga T, Baba S, Tanaka M, Kawazoe T (1990) Noninvasive estimation of the location of the end plate in the human masseter muscle using surface electromyograms with an electrode array. J Osaka Dent Univ 24, 135-140.

28. Van Eijden TM, Korfage JA, Brugman P (1997) Architecture of the human jaw-closing and jaw-opening muscles. Anat Rec 248, 464-474.
29. Widmalm SE, Lee YS, McKay DC (2007) Clinical use of qualitative electromyography in the evaluation of jaw muscle function: a practitioner's guide. Cranio 25, 63-73.

30. Spadaro A, Monaco A, Cattaneo R, Masci C, Gatto R (2010) Effect on anterior temporalis surface EMG of eyes openclosed condition. Eur J Paediatr Dent 11, 210-212.

31. Farina D, Merletti R, Indino B, Graven-Nielsen T (2004) Surface EMG crosstalk evaluated from experimental recordings and simulated signals. Reflections on crosstalk interpretation, quantification and reduction. Methods Inf Med $43,30-35$. 